\title{
A GML-Based Mobile Device Trace Monitoring System
}

\author{
Eun-Ha Song ${ }^{1}$, Sung-Kook Han ${ }^{1}$, Laurence T. Yang ${ }^{2}$, \\ Minyi Guo ${ }^{3}$, and Young-Sik Jeong ${ }^{1}$ \\ ${ }^{1}$ Department of Computer Engineering, Wonkwang University \\ 344-2 Shinyong-Dong, Iksan, 570-749, Korea \\ \{ehsong, skhan, ysjeong\} @wonkwang.ac.kr \\ ${ }^{2}$ Department of Computer Science, St. Francis Xavier University \\ Antigonish, NS, B2G 2W5, Canada \\ lyang@stfx.ca \\ ${ }^{3}$ School of Computer Science and Engineering, Aizu University \\ Aizu-Wakamatsu, Fukushima-ken 965-8580 Japan \\ minyi@u-aizu.ac.jp
}

\begin{abstract}
Recently, the demands on information services have been increasing significantly. This is mainly due to the popularization of computer and mobile telecommunication devices and the rapid improvements on wireless communication technology. Specially, information services and their corresponding management for mobile devices, such as Location Based Service (LBS) and Telematics, become more and more important. However, the standard for geographical space data has not been finalized. Many commercial monitoring systems are using their own independent geographical information without making them compatible to others. Much efforts and resources have been wasted on managing and operating those different monitoring systems' geographical Information System (GIS) databases. Accordingly, a standard format called GML, based on the most commonly used geographical data format such as DXF, DWG and SHP, has been emerged. In this paper, our work on GML's visualization in Trace Monitoring Systems (TMS) is described fully. The details on how to trace and manage data moving among different mobile terminals are presented as well.
\end{abstract}

\section{Introduction}

Geographical Information Systems (GIS) has become one of the key technology to improve and shape our future living environment, even our social life. It is an advanced information system by systematically computerizing all geographical information such as the configuration of the ground, buildings, roads, rivers, bridges, geological structure, nature of the soil, and the environment of sea. It can use these computerized data for efficiently managing national land, environment, disasters, business activities, our daily life and so on. Furthermore, the realization of wireless connectivity is bringing fundamental changes to telecommunications and computing and profoundly affects the way we compute, communicate, and interact. It provides fully distributed and ubiquitous mobile computing and communications, thus bringing 
an end to the tyranny of geography. Therefore, the mobile telecommunication industry is making the increasing efforts and investing enormous development budgets on providing high quality services on these increasingly popular mobile devices. In order to keep high quality services, such as Location Based Service (LBS), to manage trace monitoring technology on mobile devices anytime, anywhere, by any means without interruption, a trace monitoring system is very necessary. For the majority of existing trace monitoring systems on mobile devices, none of them can provide the standard geographical information because their data are based on the existing numerical map, DXF [1]. Furthermore, none of them can support and provide the integrated location information for those mobile device users. In order to overcome the above limitations, we focus on our approach on GML 3.1 [4] format which is a standard encoding language based on XML which can present a geographical and service founded on location information regardless platforms and clients. The format is very generic built on SMP (Synthetic Map Preprocessor) [5] since the existing DXF, DWG [2] SHP [3] file formats are only suitable for a special type of application. In this paper, we present our implementation approach for TMS (Trace Monitoring System of mobile devices) which can efficiently manage the integrated tracing on users' location information, after getting and saving information from the moving users who are using WIPI [6] as mobile devices.

\section{Related Works}

In this section, GML is introduced firstly, and the existing visual systems based on GML are outlined as well. Then based on the existing numerical map and other geographical formats, the mobile device monitoring systems are explained briefly.

\subsection{Map Representation Based on GML}

Due to rapid increasing demands and uses of internet or mobile telecommunications, OGC, a private GIS standard organization, offered GML 3.1 version starting from 1.0 version, to make such standard specification has interoperability of geographical information in different hardware and software environments. GML is the standard encoding language based on XML which can present a geographical and service founded on location information regardless of the kinds of platform and client. The existing visual systems based on the GML are iSMART EXplorer 4.4 [7] by eSpatial Inc., OS Master Map Viewer 2.0 [8], TatukGIS Viewer 1.4 [9] by TatukGIS Inc., FME Universal Viewer 2003 [10] by SafeSoftware Inc., and GML Viewer by ETRI.

In the following paragraph, we briefly introduce these existing visual systems one by one. We start with iSMART EXplorer 4.4 first. Its application is light, very easy to use, and has an advantage on analysis because it is connected OCI DB. And it is also possible for users to enlarge, reduce, move, and re-examine non-spatial attribute data to find out schema automatically. Among the most notable advantages, it can edit the space and offer fast response time for connecting low bandwidth through web browser. For OS Master Map Viewer 2.0 developed by JAVA, it can read 1900 points 
a second for those files in WinZip, GZip, and GML formats. It can compare and visualize their attributes after one or many spots are chosen. If some regions have a meaning, it can make them visual differently. It can change and visualize a numerical statement according to different themes. If the visual field is manipulated by mouse or keyboard (including movement, enlargement, and reduction), it will draw the visual field again after shaping the round shape of dotted line for representing the converted image. For TatukGIS Viewer 1.4, it can support almost all formats like Raster image file format and vector graphic file format and also have the capability to process the maximum $2 \mathrm{G}$ bytes. It can also convert the visual geographic information into PDF file type and measure the size of the dotted formation, the distance among positions, and the appropriate regions while handling the attributes with SQL Builder. It represents the same attributes with the equal color based on Vector attribute information. For FME Universal Viewer 2003, it shows the measuring distance, geometrical information, and the data set of the appropriate files. It is possible to distinguish each attribute with the other meaning, after saving *.fmv, to turn them into other formats. The above described visual systems can show a simple map format but they can't trace the moving course of mobile objects. Therefore, in this paper, we propose a system to trace and manage the integrated movement of mobile devices in these visual systems.

\subsection{The Existing Monitoring Systems}

The existing monitoring systems are divided into the ones with visual tracing and the ones with only raw data in tables. One typical example of the former ones is the Tracing Monitoring Solution of Cyber Map World (CyberMapWorld Co. Ltd.). It is a kind of tailored solution for tracing through GIS system to offer different applications the detailed information in real-time. Such applications include the movement course and location information of the vehicles, customers to the enterprises, government and public offices that are in need of tracing monitoring on vehicles, distribution, and customers. The system is composed of LBS engine for managing the mobile objects of the large capability and GIS engine of the technology applied with the main memory DB. It is the system to supply a solution based on LBS server included the detailed DXF files with all reduced scale. It can trace the mobile objects on an extensive scale at a time and all the vehicles and the users, and offer the searching speed out of common. The NEO controlling system, if the call is registered in the window of user's devices, indicates the call lists before the allocation, and after allocating the vehicles, the passenger's state is shown in the allocation list. If you enter the number of the car to trace, this system will show the latest location information of it based on DXF geographic information data. The managers can control the sending intervals and frequency of the data according to the states of the system.

However, for the majority of existing trace monitoring systems described above, none of them provide the standard geographical information because their data are based on the existing numerical map, DXF format. Furthermore, none of them can support and provide the integrated location information for those mobile device users. To overcome the above limitations, in this paper, we propose a system to trace 
and manage the mobile devices based on the international recommended GML standards.

\section{TMS Design}

\subsection{TMS_Server}

TMS_Server is composed of GML library module generating GML format, Server Broker controlling all TMS_Server compositions, Light-Weight Import bringing the filtering data from SMP, Moving Storage saving the information on moving space from Viewer at the time of changing the location of mobile devices, and Communication Manager communicating the mobile devices and Viewer. The architecture of these modules is described as follows in Fig. 1.

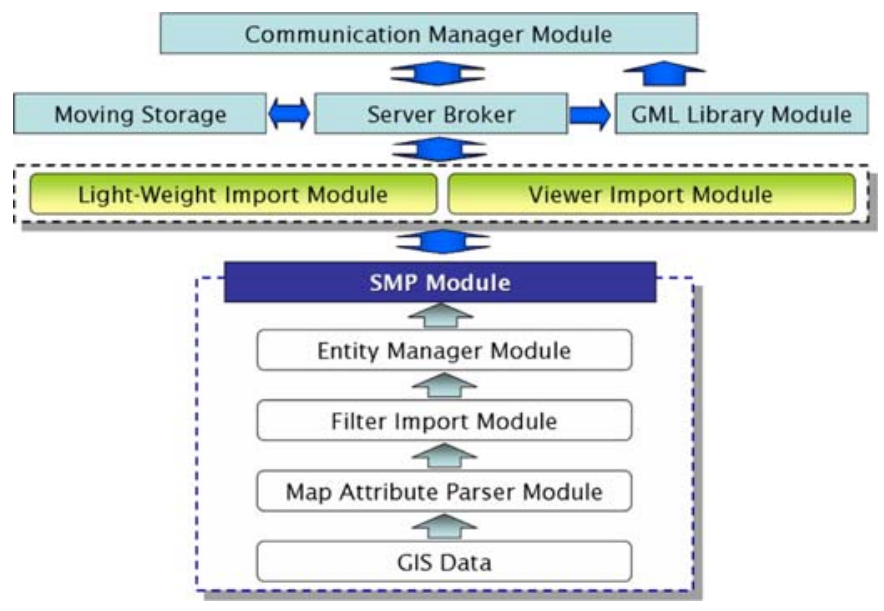

Fig. 1. Architecture of TMS_Server

\subsubsection{Light-Weight Import and Viewer Import}

Light-Weight Import considers the processing speed, the memory capacity, and the visualization area of mobile devices. Viewer Import plays a major role of supporting monitoring based on the standard geographic files. The former module extracts layer and attributed entity section from the source geographic information data such as DXF, DWG, and SHP files at SMP, and saves as the light-weighted files. The latter module saves the extracted files. After that, these files are returned into manipulation map files and memorized in each memory address.

Checking the memorized files, the scale map sheet code building files are stored in theme which are indicated as layer 4111=Building, 4112= Glasshouse, 4113=Historic Interest, on the other in term, these are stored in outline as level 50. In the scale map sheet code road, theme is stored as land, group as road or track, 
term as public with level 50. These data are also stored without handling the attributes specially.

\subsubsection{GML Library Module}

GML library module controls the converting process, which changes the geographic information data from Server Broker into the geographic space information data with the same type of GML 3.1 format based on XML. The detailed contents in each class of GML library module are listed in Table 1.

Table 1. Classes of GML library module

\begin{tabular}{|l|l|}
\hline \multicolumn{1}{|c|}{ Class } & \multicolumn{1}{c|}{ Description } \\
\hline \hline AnchorPoint & Selecting a fixed GML point \\
\hline CartographicMember & To make CartographicText \\
\hline CartographicText & To save the assigning attributes in showing Text \\
\hline CoordinateTuple & To make AnchorPoint (Punctual Coordinates) \\
\hline Feature & To establish a basic model(fid =10000000, FeatureCode=0) \\
\hline FeatureCollection & Integrating CartographicMember and TopographicMember \\
\hline GMLDocument & $\begin{array}{l}\text { Showing text, polyline(attributes, coordinates), } \\
\text { polygon(attributes, coordinates), point }\end{array}$ \\
\hline GMLMulPoint & Accepting various CoordinateTuple with GML point \\
\hline GMLPoint & To make CoordinateTuple \\
\hline GMLSchema & $\begin{array}{l}\text { To make GML documents (parsing order: text-polygon- } \\
\text { polyline) }\end{array}$ \\
\hline GMLSchemaImp & Defining tag of GML documents \\
\hline TextRendering & Defining text description \\
\hline TextString & To description of letters line \\
\hline Topographic & Defining the attributes to be included Topographic point \\
\hline TopographicMember & Defining each attribute to be included Topographic \\
\hline
\end{tabular}

\subsubsection{Communication Manager}

Communication Manager is in charge of the communications among the WIPI users, TMS_Server, and Viewer. This module delivers the geographic data of the lightweighted GML 3.1 format from the server to Viewer through UDP communications, the mobile devices, and TCP/IP protocols. With the present coordinates as its starting points, it delivers the geographic data as the size of $250 \times 250$ to show the size $120 \times 146$ as a visualization area in mobile devices. It delivers the geographic data at the shape size of $2500 \times 2500$ than the size in the appropriate map sheet to Viewer. This module also saves and delivers moving information from the mobile devices a second. If Viewer wants to know the geographic space information enters its ID, this module gives the moving information in mobile devices to TMS system. Table 2 shows each class file in Communication Manager. 
Table 2. Classes of Communication Manager

\begin{tabular}{|c|l|}
\hline Class & \multicolumn{1}{|c|}{ Description } \\
\hline \hline ReadThread & Disconnecting and delivering the read data \\
\hline ServerConn & $\begin{array}{l}\text { Recording the connected time, date, file size in server, perform- } \\
\text { ing GML creating, handling the original data file, and deliver- } \\
\text { ing created GML data }\end{array}$ \\
\hline SimpleServer & Indicating the comments in GML server \\
\hline
\end{tabular}

\subsubsection{Server Broker}

Server Broker is a kind of medium coordinating and controlling each module. These class files are shown in Table 3. It demands GML file corresponding to the coordinates moving in mobile devices, and has a role of delivering the coordinates to the basic coordinates of GML library module. It receives the ID's moving information from mobile devices in Viewer, delivers this moving information to Communication Manager, and sends the coordinates to the core ones of GML library module for the initial visualization.

Table 3. Classes of Server Broker

\begin{tabular}{|l|l|}
\hline \multicolumn{1}{|c|}{ Class } & \multicolumn{1}{|c|}{ Description } \\
\hline MapPatcher & $\begin{array}{l}\text { Recording the connected time, date, file size, performing } \\
\text { GML creating in server, if making errors, recording "The phone } \\
\text { is off", adding method to short type converting byte[] }\end{array}$ \\
\hline Mapserver & $\begin{array}{l}\text { Allowing Socket approaching, if making errors, recording "This } \\
\text { is already used port or security port" }\end{array}$ \\
\hline MapServerImp & Designating numbers of IP and port \\
\hline
\end{tabular}

\subsubsection{Moving Storage}

Moving Storage is a repository with the moving information in mobile devices according to ID through Server Broker. This stored data is returning TM coordinates into text.

\subsection{Viewer}

Viewer is composed of Communication Manager module corresponding with TMS_Server in Fig.2. Server Broker is used for mediating and adjusting all viewers and handling the coordinates, GML parser is used for analyzing GML file into XML file and dividing according to each attribute, User Event is used for handling event message when manager of Viewer moves the map, Monitoring Event module is used for tracing and managing the moving information in mobile devices for monitoring.

\subsubsection{Communication Manager}

Communication Manager receives the geographic data of GML format from TMS_Server through TCP/IP protocols and the moving space information about 


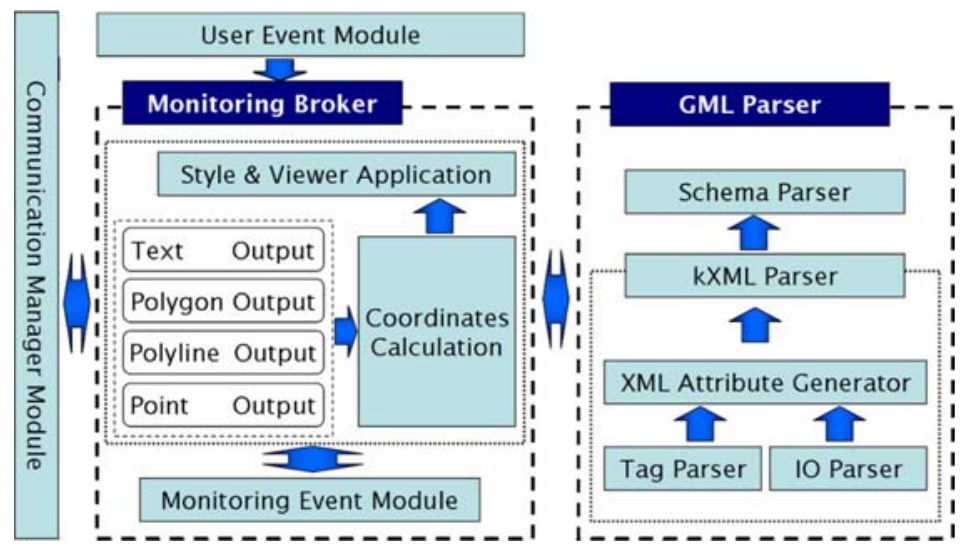

Fig. 2. Architecture of TMS Viewer

mobile device ID's shifting of mobile devices from TMS_Server. In details, when there is demand on the event from User Event, this module will send and receive the geographic space information through communicating with TMS_Server.

\subsubsection{GML Parser}

GML parser is a module of analyzing GML file for the visualization. GML is based on XML, and this paper adopted GML 3.1 format version. There are feature schema, geometry schema, GML schema, and application schema. After distinguishing every schema in schema parser, the relevant schema has a charge of working and sorting tag and contents as the attributes of XML is sorted in kXML Parser. Then it moves contents matching the sorted tag in pair to Monitoring Broker.

\subsubsection{User Event}

User Event manages the event messages demanded from Viewer such as enlargement, reduction, and manual movement of map to a specified area.

\subsubsection{Monitoring Broker}

Monitoring Broker is a module of adjusting and mediating the geographic information among modules. It delivers the geographic space information from TMS_Server to GML parser, after receiving the analyzed geographic space information from $G M L$ parser, to make these data show to Viewer, adding the matching coordinates and applied the styles to text, polygon, polyline, and point.

\subsubsection{Monitoring Event}

Monitoring Event makes the continuous changing space coordinates visible to Viewer. Because the locations in mobile devices change every moment, the changing coordinates in the geographic space saved to mobile devices' ID make them visible at once a second in Monitoring Broker. 


\section{Implementation and Case Studies}

The implementation of TMS uses JAVA 1.3.1-b24, JBuilder 2005 as the programming tool. The implemented mobile devices, because of limitations in using the practical devices, are AROMA-WIPI Emulator Evaluation Ver. 1.1.2.7 [14]. The visualization of viewer adopted the area of map sheet 4_35701077 and the address is as the followed; $3 \mathrm{Ga}$, Hanok Village, Pungnam dong, Wansan gu, Jeonju, Jeonbuk, 560-033 Korea.

In Fig. 3, the mobile device ID 1 after joining in Viewer moves from Jungang elementary school to Chongsu pharmacy. In Fig. 4, the mobile device ID 2 moves from Jeonju tradition museum to Sungmun church. In Fig. 5, the mobile device ID 3 moves from Gyodong temple of Wonbuddism to piano academy. Fig. 6 is a console screen of

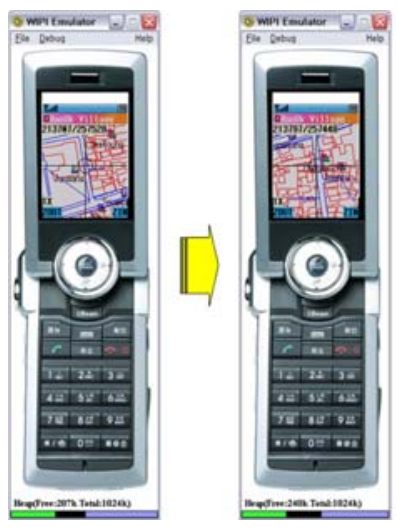

Fig. 3. Mobile Device ID 1

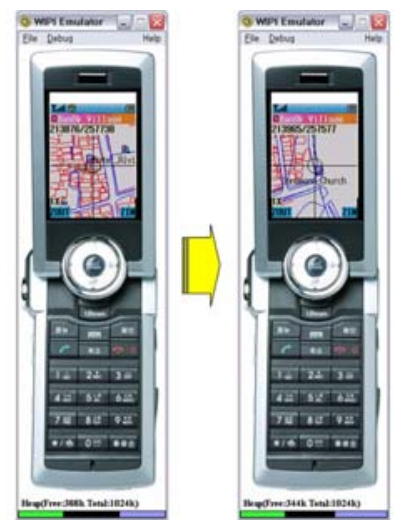

Fig. 4. Mobile Device ID 2

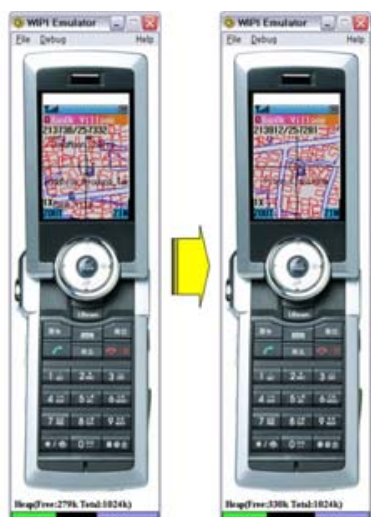

Fig. 5. Mobile Device ID 3

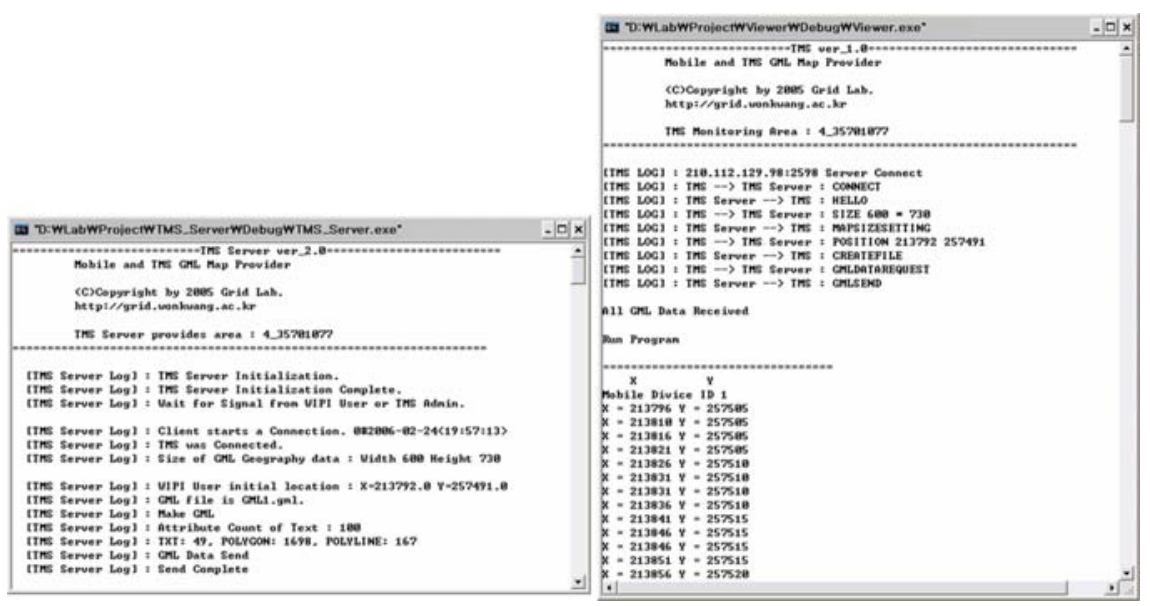

Fig. 6. TMS console of map sheet 4_35701077 


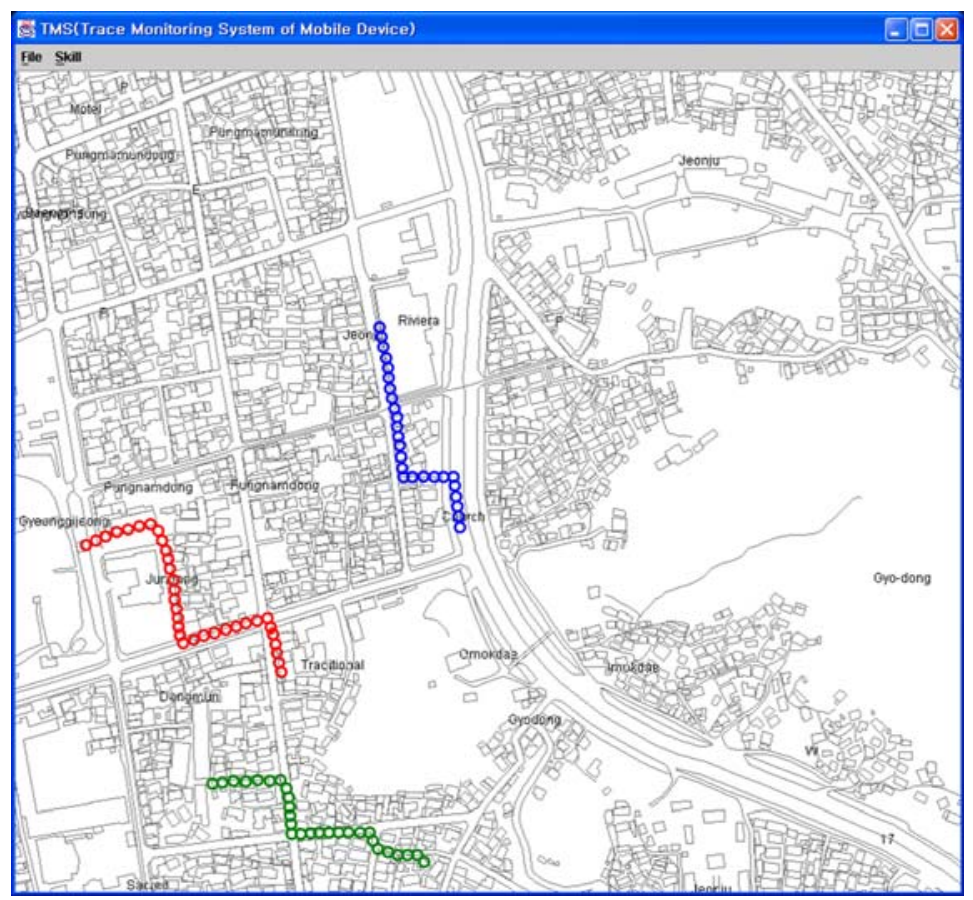

Fig. 7. Viewer of map sheet 4_35701077

TMS_Server offering the geographic information to Viewer, and a console screen of TMS recorded with the moving coordinated in the mobile device ID 1 and received the geographic information from TMS_Server. In Fig. 7, the mobile ID 1 is shown with blue color, the mobile ID 2 with red color, and the mobile ID 3 with green color.

\section{Conclusions and Future Works}

In order to import DXF, DWG, SHP file as a basic numerical map files easily in TMS, in this paper we filter and exclude the unnecessary parts in the existing format visible according to the tag and the attributes. Then, to import the filtered file based on SMP module capable of composing the basic geographic information of TMS system rapidly and easily, the scale and map sheet is stored as clear numbers and each file can be made to manage uniquely. TMS is implemented to make it visible based on GML 3.1 format suggested in OGC, to attain TM coordinates as the real time moving space information of various mobile devices, and to make it possible to get the integrated tracing management sending these to TMS_Server.

In this paper, the implemented TMS is applied to the existing TMS to the present call systems without any special equipment because it can, on the manager's part, trace and manage the user's mobile device. In using the geographic information, it makes GML format adopted the international geographic recommended standard instead of the present numerical map for visualization. As for the numerical map, to 
make GML format, it makes the unnecessary information filtered and light-weighted, and offers indexing files.

In future work, it is necessary to develop TMS service which make use of the public DXF which is popular geographic information service and SVG getting vector graphic easily in the internet environment, to apply DEM (Digital Elevation Model) as an actual measured topographic data to represent the real space information, and to apply LOD (Level of Detail) as the brief handling technology on all areas classifying the far and near and controlling the degree of accuracy. And it is also necessary to develop TMS to indicate only location on map which the users as multimedia thematic map want making use of the delivery of meaning the merits of GML 3.1, and adding POI (Point of Interest) information in each main building to the attribute information.

\section{Acknowledgement}

This work was supported by the Korea Research Foundation Grant funded by the Korean Government (MOEHRD) (the Center for Healthcare Technology Development, Chonbuk National University, Jeonju 561-756, the Republic of Korea).

\section{References}

1. Autodesk Drawing eXchange Format, http://www.autodesk.com/techpubs/autocad/ acad2000/dxf

2. Open Design Alliance, http://www.opendwg.org

3. ESRI, ESRI Shapefile Technical Description. ESRI, INC, http://www.esri.com, 1998.

4. OpenGIS Consortium, Inc. Geography Markup Language (GML) Implementation Specification, http://www.opengeospatial.org

5. C-Y Jeon, J. Park, E-H Song, Y-S. Jeong, “A Development of Integrated Map Preprocessor for Mobile GIS Visualization,” KIPS, VOL.12 NO.1 pp. 707 710, 2005.

6. Wireless Internet Standardization Forum, "Mobile Standard Platform WIPI 2.0.1", http://wip i.or.kr, 2004.

7. eSpatial Inc. iSMART Explorer 4.4, http://www.espatial.com

8. Snowflake Software Ltd. OS Master Map Viewer 2.0, http://www.snowflakesoftwa re.co.uk

9. TatukGIS Inc. TatukGIS Viewer 1.4, http://www.tatukgis.com/

10. Safe Software Inc. FME Universal Viewer 2003, http://www.safe.com

11. CyberMapWorld co., Location Tracing Solution, http://lbs.cybermap.co.kr/

12. Canas C\&C co., http://www.kindcall.com/

13. kXML, http://kxml.kobjects.org/

14. Aromasoft co., AROMA-WIPI Emulator Evaluation, http://www.aromasoft.com 